\title{
Current and future applications of probiotics
}

\section{Maria Carmen Collado, Miguel Gueimonde, Gaspar Pérez-Martínez}

$5{ }^{1}$ Instituto de Agroquímica y Tecnología de Alimentos (IATA-CSIC), Valencia, Spain.

${ }^{2}$ Instituto de Productos Lacteos de Asturias (IPLA-CSIC), Asturias, Spain.

\begin{abstract}
10 Dysfunctions of the gastrointestinal tract are thought to be related to disturbances or aberrancies of the intestinal microbiota. Nowadays probiotics may represent a solution of choice to balance gut microbiota although they have not been selected for specific subpopulations or disease groups. Since probiotic health benefits are strain specific, it is likely that strains can be selected aimed at particular groups of patients.

15 Probiotics have been usually used to treat and prevent some gastrointestinal disturbances such as irritable bowel diseases (IBD) or syndrome (IBS), or diarrhoea, although new extra-intestinal applications are the getting interest of industry and consumers. This review shows the current and new applications of probiotics to improve human health.
\end{abstract}

Key words: probiotic, microbiota, disease 


\section{Introduction}

Gastrointestinal tract (GIT) microbiota plays an important role in human health due to its essential participation in digestion and nutrient assimilation, as well as its immune modulating functions. The populations of bacterial species in the gut vary between

5 individuals, they are influenced by the environment and, although flexible, microbial populations in the gut are finely tuned and very sensitive to infectious processes (Kurokawa et al., 2007; Palmer et al., 2007). Also, the GIT microbial profile varies in immunological or inflammatory disease patients, metabolic syndrome and even in children with autism (Sekirov et al., 2010). Furthermore, recent findings suggest that

10 specific gut microbial imbalances are associated with enhanced risks of specific diseases. A sensible option to restore the lost balance in a complex microbial community may be the introduction of bacterial species (probiotics) that will restitute it. Probiotics are defined as "live microorganisms that, when administered in adequate amounts, confer a health benefit to the host" (FAO/WHO, 2002). They are commonly

15 isolated from human samples and are externally administrated helping in the recovery and maintenance of the microbial balance. Probiotics have become popular in a number of food products which are commercially available worldwide. They are also found with increasing frequency as nutritional supplements, freeze dried in sacchettes or capsules. Most of them belong to the genera Lactobacillus or Bifidobacterium, although there are

20 remarkable exceptions, such as Saccharomyces boulardii (McFarland et al., 1995), Escherichia coli Nissle 1917 (Kruis et al., 1997) or some Bacillus species (Duc et al., 2004; Hong et al., 2008).

During the last ten years, health and wellbeing have been one of the strongest market drivers for the food industry. Therefore, national and supranational regulatory agencies have intervened to warrant the proper use of the terms and concepts also demanding 
solid scientific evidence for any health claim used in functional foods such as probiotics (http://nccam.nih.gov/health/probiotics/; EU Reglament 1924/2006EU). This has reinforced research and remarkable advances have been achieved in our knowledge on targets and modes of action. There are hundreds of scientific publications describing 5 different effects of the probiotic intake in humans, then, meta-analyses and reviews evaluated the level of scientific evidence for clinical use attained by those studies (Gill and Guarner, 2004; Williams, 2010). Hence, from the clinical perspective, solid scientific evidences on their beneficial effects have made probiotics more and more frequently prescribed in Modern Medicine.

10 The mechanisms of action of probiotics are mostly unknown, however, they must be necessarily related to their observed effects, and as such, they can be grouped in: antiinfective (treatment or prevention of viral, yeast or bacteria pathogenesis), metabolic functions (amelioration of lactose malabsorption and lowering blood cholesterol) and modulation of the immune response system (improvement of immune response, 15 amelioration of inflammatory syndromes and immune related pathologies). The antiinfective effects of probiotic bacteria involve mechanisms that imply antagonism with other bacteria, or competitive binding to receptors, they can also establish an effective communication through biochemical signals with intestinal epithelial cells or mucosa, inducing local defense substances (i.e., IgA) in the mucosa (Corr et al., 2009; Ohland 20 and MacNaughton, 2010), homeostasis or epithelial integrity (Bäuerl et al., 2011; Di et al., 2005; van Baarlen et al., 2009; van Baarlen et al., 2010). Those interactions may partially explain the mechanisms used by probiotics to improve immune dysfunctions, where a wide research field has just been opened. However, the study mechanisms of host-probiotic interactions show difficulties that surpass most modern techniques utilized. In particular, their interaction with GALT depends upon multiple receptors 
that, altogether elicit different and complex signal transduction pathways. Understanding the mechanisms of action of probiotics will help to substantiate Health Claims for commercial purposes, but most important, it will explain differences between strains and will help to define more precisely all aspect of their health benefits.

5 This review is aimed to compile representative scientific evidence showing the great diversity of applications of probiotics and their future perspectives.

\section{Benefits of probiotics on human health}

This far the lack of a homogeneous regulation has led to the coexistence in the market

10 of well studied strains for which health benefits have been demonstrated with others for which not scientific data are available. The new EU regulation on nutrition and health claims made on foods has come into force (Regulation (EC) $\left.\mathrm{n}^{\circ} 1924 / 2006\right)$ which may help to increase research efforts to scientifically substantiate probiotic effects.

Some of the beneficial effect of probiotic consumption include, among others:

15 improvement of intestinal tract health by means of regulation of microbiota and stimulation and development of the immune system; synthesizing and enhancing the bioavailability of nutrients; reducing symptoms of lactose intolerance and reducing risk of certain diseases. Several mechanisms of action have been proposed to explain the beneficial effects of probiotics. These mechanisms are largely unknown, but may

20 involve modifying gut $\mathrm{pH}$, antagonizing pathogens through production of antimicrobial compounds, competing for pathogen binding, receptor sites, nutrients and growth factors, stimulating immunomodulatory cells, and producing lactase [ref].

Probiotics have specific targets of action that are probiotic-dependent as different bacterial strains may differ with respect to their effects on health. In addition, it must be taken into consideration that these mechanisms may be multi-factorial processes and 
also, dependent on each probiotic strain that may have specific functions affecting to the health host. Considering their possible targets, probiotics would be designed and applied in specific diseases based on their characteristics and health-promoting effects. Different levels of host-microbe interaction can be distinguished: i) bacteria-gut

5 epithelium interaction: adhesion to mucosal and epithelial cells, stimulation of mucus secretion and enhancing the production of defensive molecules such as mucins and defensins, increasing and reinforcing barrier function, innate immune function, reducing the secretory and inflammatory consequences of bacterial infection and improving gut motility; ii) bacteria-immune system interaction: Immune-modulation and regulation

10 of immune responses beyond the gut and finally, iii) bacteria-bacteria interaction: Modify microbiota to suppress and inhibit pathogens and prevention of their adhesion, establishment and/or replication of pathogens in the gastrointestinal tract, secretion of antimicrobial substances and compounds, compete for nutrients necessary for pathogen survival and anti-toxin effects. The general mechanisms by which probiotics may have 15 an effect can be divided into three main categories: normalization of microbiota, modulation of immune response, and metabolic functions.

Traditionally probiotics have been tested for several different potential benefits, in the hope that the strain will have some of the tested effects. However, since probiotic health benefits are strain specific, it is likely that strains can be selected aimed at particular

20 groups of consumers. Also particular patient groups may require specific probiotics strains aimed at the groups' particular needs. In addition to efficacy, an assessment of the cost and need for routine use of probiotics should also be conducted in order to identify those areas where probiotics are more likely to be used and they may have a deeper effect. Therefore, an alternative would be to rationally select strains for a specific health benefit. In spite of the lack of scientific rationale on the traditional 
selection procedures, the results of human clinical studies have produced good evidence on the health effects of specific probiotic strains. However, many other proposed health effects still require rigorous clinical studies to prove their health promoting potential. As the use of specially selected new probiotics to maintain health must be considered even

5 more promising, the selection criteria need to be revised.

\section{Current Intestinal Applications}

Probiotics have been traditionally used in cases of intestinal discomfort. It has been known for a long time that fermented dairy products containing live bacteria are better

10 tolerated than milk by lactose intolerant subjects. Among the current intestinal applications of probiotics the beneficial effects of specific strains shortening the duration and preventing the episodes of diarrhoea are the best documented. Interestingly, their effect on constipation has also been evaluated. The role of probiotics on inflammatory bowel disease and irritable bowel syndrome has been the subject of

15 several recent studies. During the last years probiotic beneficial effects on Helicobacter pylori infection, colorectal cancer or necrotizing enterocolitis have also been assessed. Some beneficial intestinal effects of certain probiotic strains can be considered well established which has lead to the publication of recommendations for their use in clinical practice (Floch et al. 2008).

\section{Lactose intolerance}

A significant part of the population shows reduced levels of $\beta$-galactosidase in the small intestine, suffering intolerance symptoms when lactose is ingested. Several studies have shown that lactose-intolerant subjects suffer fewer symptoms if milk is replaced by

25 fermented dairy products. The bacterial enzyme $\beta$-galactosidase, which can be detected 
in the small intestine after consumption of viable yoghourt, appears to be the major factor improving digestibility by hydrolysing the lactose. It is important to underline that different strains may have very different $\beta$-galactosidase activities and thus differ in their efficacy. Another factor suggested to have a role is the slower gastric emptying of

5 semi-solid milk products compared with milk. Modulation of the intestinal microbiota composition and activity may also play a role alleviating lactose intolerance (He et al. 2008).

\section{Diarrhoea}

10 Diarrhoea is an obvious target for probiotics and their role on infant diarrhoea is the best studied probiotic effect. Several human intervention studies indicate a clear positive effect of specific probiotic strains on both, rotavirus and antibiotic-associated diarrhoea. In fact, probiotics are considered among the alternatives for management of acute gastroenteritis in children (Guarino et al. 2008). Results for traveller's diarrhoea,

15 although encouraging, are less convincing. As with any other probitotic effect the beneficial effects reported in the studies are specific to the strains used.

Roravirus diarrhoea. Shortening the duration of rotavirus diarrhoea using specific probiotic strains is well documented. Several studies around the world reported 20 beneficial effects (Kotowska et al. 2005, Weizman et al. 2005, Szymanski et al. 2006). Some meta-analyses have evaluated the effects of probiotics in general concluding that probiotics reduce the duration or frequency of diarrhoea in children (Huang et al. 2002, Van Niel et al. 2002, Allen et al. 2004). The main criticism to these meta-analyses is that often they considered probiotics as a group. Different probiotics have varying 25 effects on the duration of diarrhoea and the immune response in children with acute 
gastroenteritis. Thus, it is important to remember that the reported studies are specific to the strains used. The increasing number of studies conducted during the last years allowed to carry out meta-analyses focused on specific strains. These studies showed that, at least, the strains Lactobacillus rhamnosus GG and $S$. boulardii are effective in 5 treatment of acute diarrhoea in children (Sazawal et al. 2006, Szajewska et al. 2007a, b).

Antibiotic-associated diarrhoea. The efficacy of probiotics reducing this common kind of diarrhoea has been extensively studied and the results are convincing for a number of probiotics. Meta-analyses conducted in this area (Sazawal et al. 2006, Johnston et al. 10 2007) concluded that the available data provides sufficient evidence for the role of probiotics in the prevention of antibiotic-associated diarrhoea. For some specific probiotics such as L. rhamnosus GG and $S$. boulardi strain-focused meta-analysis have also indicated a beneficial effect of the strains (McFarland 2006, Szajewska et al. 2006).

15 Traveller's diarrhoea. There are a few studies reporting a positive effect of probiotics for reducing the risk of traveller's diarrhoea . However, inconsistent results have often been reported with unexplained geographic differences in protection rates (Koo and Dupont 2006). The different aetiology of the disease depending on the destination and differences in the probiotic strains, dosages and administration vehicles used makes the comparison of studies difficult. A recent meta-analysis reports a beneficial effect of certain strains in the prevention of traveller's diarrhoea (McFarland 2007). However, information on large-scale studies using defined strains is still lacking. 


\section{Inflammatory Bowel Disease}

Crohn's disease, ulcerative colitis (UC) and pouchitis constitute a group of diseases whose aetiologies are not well understood and that are characterised by deregulation of the immune system leading to gut inflammation. This group of diseases is commonly

5 regarded as inflammatory bowel disease (IBD). A disturbance of gut microbiota or an inadequate host response to it has been shown to play a role in the pathogenesis of IBD (Manichanh et al. 2006; Qin et al. 2010). The effect of different probiotics on IBD has been evaluated with variable results.

The greatest evidence for efficacy of probiotics on IBD has been shown for the

10 prevention of pouchitis. Different human intervention trials have shown effectiveness of the probiotic preparation VSL3 in maintaining remission of pouchitis (Gionchetti et al. 2003, Mimura et al. 2004). A non-pathogenic E. coli strain (Nissle 1917) was shown to be as effective as the standard treatment (masalazine) in maintaining remission of UC (Kruis et al. 2004) and the mix VSL3 was suggested to induce remission and to improve

15 status in UC patients (Bibiloni et al. 2005), supporting the use of probiotics. $L$. rhamnosus GG was found to prolonge the relapse-free time, although no effect on relapse rate was observed (Zocco et al. 2006) and some positive results have also been reported for S. boulardi (Guslandi et al. 2003). In contrast, trials evaluating the application of different probiotics on Crohn's disease have not been too successful

20 (Prantera et al. 2002, Marteau et al. 2006) and only some preliminary studies reported modest beneficial effects (Guslandi et al. 2000, Guandalini et al. 2002).

Some reviews and meta-analysis studies (Elahi et al. 2007, Mallon et al. 2007) focused on the use of probiotics on IBD concluded that further research is still needed. 
Irritable Bowel Syndrome (IBS) affects a significant part of the western population being the first cause of visit to the gastroenterologist. IBS is characterised by intestinal discomfort or pain and altered bowel function with diarrhoea and constipation. Although the aetiology if the disease is not well understood, in IBD patients an altered

5 brain response to visceral stimuli appears to occur (Naliboff et al. 2001) causing pain or discomfort due to visceral hypersensitivity. An altered gut microbiota has also been reported in IBS patients (Nobaek et al. 2000, Pimentel at al. 2000) providing the basis for probiotic theraphy. In a study using Lactobacillus plantarum $299 \mathrm{v}$ a reduction of symptoms was observed (Niedzelin et al. 2001) and reductions of bloating and

10 flatulence have also been reported for the probiotic mix VSL3 (Kim et al. 2003, 2005). However, the limited number of individuals included in these studies requires further confirmation in larger trials. Contrary to this no reduction of symptoms was observed with other strains (Drouault-Holowacz et al. 2008). Recently it has been shown that oral administration of specific Lactobacillus strains induces the expression of opioid and cannabinoid receptors in intestinal epithelial cells (Rousseaux et al. 2007). Such reaction mediates gut analgesic effects indicating that the gut microbiota composition and its modulation through probiotics can influence our visceral perception which constitutes a new approach to modulate the manifested visceral hypersensitivity of IBD patients.

20 During the last years large studies have been conducted reporting beneficial effects with some strains and probiotic mixes (Kajander et al. 2008, Whorwell et al. 2006, Guyonnet et al. 2007). A recent meta-analysis concludes that although some specific strains or mixes may have a beneficial effect further large well designed studies are needed (Mcfarland and Dublin 2008). 


\section{Helicobacter pylori therapy}

$H$. pylori is a worldwide spread infectious agent that colonizes the stomach causing gastritis, peptic ulcer and gastric cancer. Animal and human studies have reported the inhibitory effect of certain lactobacilli preventing infection and colonization (Coconnier

5 et al. 1998, Wang et al. 2004) or reducing bacterial load (Francavilla et al. 2008). A good in vitro inhibition was observed for Lactobacillus johnsonii La1 and the strain has positive effects when consumed during eradication therapy (Felley et al. 2001). In a multicentre study the consumption of a fermented milk containing Lactobacillus casei DN-114001 increases the eradication efficacy of the standard treatment in children

10 (Sykora et al. 2005). Similar results have been reported in adults with a probiotic yoghourt (Kim et al. 2008). Furthermore, probiotics may have other beneficial effects in the setting of $H$. pylori antibiotic therapy by alleviating the side-effects associated with the standard antibiotic treatment (Myllyluoma et al. 2005, Cindoruk et al. 2007). Gotteland and coworkers (2006) reviewed this topic concluding that probiotics may not

15 be effective eradicating $H$. pylori but in combination with the standard treatment they may increase eradication rates and/or reduce side-effects.

\section{Constipation}

Constipation is a common intestinal disorder due to deregulation of the motor activity in

20 the large intestine that affects a significant part of the western population. Probiotics have been suggested to relieve constipation and LAB-containing fermented products have been used to this end. The strains B. lactis DN-173 010, L. casei Shirota or L. johnsonii La1 have been reported to be beneficial (Marteau et al. 2002, Koebnick et al. 2003, Fukushima et al. 2004). 


\section{Necrotizing enterocolitis (NEC)}

Recent studies suggest that certain probiotics can reduce both the severity and incidence of NEC in preterm neonates (Hoyos 1999, Bin-Nun et al. 2005, Lin et al. 2005) whilst no effect has been observed with other probiotic strains (Dani et al. 2002), underlining

5 the high strain-specificity of probiotics. A meta-analysis study concluded that probiotics may be effective in reducing the risk of NEC in low-birth weight infants but more research is needed to clarify their safety, appropriate dose and type of probiotic to be used (Deshpande et al. 2007).

\section{Colorectal cancer}

Colorectal cancer is the third most common cancer and it seems to be strongly linked to dietary habits (Bingham et al. 2003). The association between gut microbiota composition and risk of colon cancer has been a subject of discussion for many years (Moore and Moore 1995, Hope et al. 2005). Some reports appear to indicate that not

15 just the faecal microbiota but the colonic mucosa microbiota may be altered in these patients (Gueimonde et al. 2007). A potential role for probiotics in the prevention of this form of cancer has often been suggested. However most of the available evidence is indirect being based on in vitro and animal studies. There are also some case-control studies supporting an inverse relationship between consumption of yoghourt and risk of colon cancer (Rowland 2004). However, long-term trials as well as epidemiological studies are needed before any conclusion may be established.

\section{Current Extra-Intestinal Applications}




\section{Urinary tract and vaginal infections}

A healthy vaginal microbiota is characteristically dominated by lactobacilli, which are known to provide colonization resistance and prevent the infections by means of different mechanisms including: specific adhesion sites at the epithelial surface of the

5 urinary tract; $\mathrm{pH}$ control and the production of antimicrobial substances like acids, hydrogen peroxide and bacteriocines; degradation of polyamines; and also, production of surfactants, which have anti-adhesive properties against the adhesion of pathogens (; Boskey et al., 1999; Aroucheva et al., 2001; Reid, 2001). The balance can be disturbed to a complex multispecies microbiota including the overgrowth of indigenous bacteria

10 of the vagina like Gardnerella, Bacteroides, Peptostreptococcus, Prevotella spp. or aerobic cocci, or by the invasion of foreign microorganisms, such as E. coli, Enterococcus faecalis, Enterobacteriaceae, staphylococci or Candida, may lead to urinary tract infections and bacterial vaginosis (Xie et al., 2006; Fredriks et al., 2005; Reid et al., 2008; Kirvajainen et al., 2009).

15 Some Lactobacillus including strains of L. gasseri, L. acidophilus and L. salivarius have been found to have an inhibitory effect on the in-vitro growth of pathogens such as $G$. vaginalis, Bacteroides spp., or Prevotella bivia that may cause infections (Mastromarino et al. 2002; McLean and Rosenstein 2000). Production of lactic acid by lactobacilli, which is mainly responsible for the low vaginal $\mathrm{pH}$, contributes to the inhibition of

20 growth of $G$. vaginalis. In addition, production of bacteriocins by some Lactobacillus strains has also been found to play a role in the inhibition of growth of G. vaginalis (Aroutcheva et al., 1991; Simoes et al., 2001). Some in-vitro studies have also shown that specific strains of lactobacilli are able to aggregate with $G$. vaginalis and inhibit the adherence and/or to displace previously adhered pathogens from vaginal epithelial cells

25 (Boris et al., 1998; Mastromarino et al., 2002). Most relevant clinical trials have 
suggested that oral administration of lactobacilli, is able to increase the numbers of vaginal Lactobacillus, restore the vaginal microbiota to normal, and treat women suffering bacterial vaginosis, although some trials found that intra-vaginal administration had no significant effect (Falagas et al., 2007). Although the high

5 number of available studies and results concerning the effectiveness of the administration of lactobacilli for the treatment of bacterial vaginosis are mostly positive, its needed further studies to conclude definitively that probiotics are useful (Falagas e al., 2007).

\section{Allergic diseases (Atopic disease, eczema and rhinitis and asthma)}

The prevalence of allergic diseases continues to increase in the developed world and constitutes a common health problem among children (Asher et al. 2006; von Mutius 1998). Atopic disease develops in the first years of life, and other allergic processes can gradually manifest over time. The condition usually manifests initially in the form of

15 food allergy and atopic dermatitis, followed in later stages by other problems as respiratory allergy with rhinitis and/or asthma. This has led to the adoption of preventive measures in those nursing infants at highest risk of developing atopy. However, these are very complex diseases involving the intervention of a broad range of etiopathogenic factors: environmental, genetic and epigenetic. Microbiota aberrancies

20 have been related to the development of allergic diseases (Kalliomaki et al., 2001; Kalliomaki and Isolauri, 2003). A reduced ratio of bifidobacteria to clostridia has appeared in infants developing atopy (Kalliomäki et al. 2001), and allergic patients have been shown to be more often colonized with Clostridium and Staphylococcus, and have fewer Enterococcus and Bifidobacterium than do non-allergic patients (Björksten et al. 
precede the development and manifestation of atopic outcomes, while early colonisation with Escherichia coli has been associated with higher risk for developing eczema, and Clostridium difficile with eczema, recurrent wheeze, and allergic sensitization in infancy (Penders et al. 2007). The Hygiene Hypothesis supports the rapid increase of 5 atopy related to lower microbe exposure at early life and subsequent lower number of infections during infancy (Strachan, 1989). These changes on microbiota may be counter-balanced by probiotic bacteria, and also, positive clinical effects on the prevention and treatment of atopic diseases of probiotics have been reported (Majamaa and Isolauri 1997; Isolauri et al. 2000; Kalliomäki et al. 2001b, 2003; Rosenfeldt et al.

10 2003; Viljanen et al. 2005b; Abrahamsson et al. 2007).The effectiveness of L. rhamnosus GG in the prevention of atopic dermatitis has been reported in randomized, controlled trials (Kalliomaki et al., 2001; 2003). L. rhamnosus GG was given to pregnant women for four weeks prior to delivery, then to newborns at high risk of allergy for six months with the result that there was a significant reduction in early 15 atopic disease (Kalliomaki, 2003). In other clinical studies with infants allergic to cow's milk and also, atopic dermatitis was alleviated by ingestion of probiotic strains $L$. rhamnosus GG and B. lactis Bb12 (Majamaa and Isolauri, 1996; 1997; Isolauri et al., 2000). Furthermore, several well-designed studies have provided evidence that specific strains of probiotics can be somewhat effective in treatment of established atopic 20 dermatitis (Rosenfeldt et al. 2003; Viljanen et al. 2005; Weston et al. 2005). In other studies, L. rhamnosus GG was combinated with other probiotic strains, L. rhamnosus LC705, B. breve Bb99, and Propionibacterium freudenreichii subsp. shermanii JS in order to determine the impact on the cumulative incidence of allergic diseases (Kukkonen et al. 2007). This study showed no effect on incidence of all allergic 25 diseases, but the treatment did significantly prevent atopic eczema. Two studies 
showed a preventive effect of probiotic supplementation on the development of eczema at the age of 2 years, with a relative risk reduction of 50\% (Kalliomaki et al., 2001 ) and $26 \%$ (Kukkonen et al., 2007). In a third study, the cumulative incidence of eczema was similar in intervention and placebo group, but less atopic eczema was observed in the

5 intervention group (Abrahamsson et al., 2007). In two studies, supplementation of probiotic bacteria failed to reduce the risk of atopic dermatitis and resulted in increased sensitization to allergens in one study (Taylor et al., 2007; Kopp et al., 2008). A major reason for these discrepant clinical results may be the use of different probiotic strains or preparations. In this respect, however, it is interesting that the results of Kopp et al.

10 (2008) are in sharp contrast with the study of Kalliomaki et al., (2001 lancet) although an identical probiotic strain is used (LGG) in an also otherwise comparable study design. Other study (Niers et al., 2009) indicates that the beneficial effects of probiotic bacteria are established within the first 3 months of life. It reported a relative risk reduction of $58 \%$ of parental reported eczema at 3 months of age being the effect 15 sustained until the age of 2 years, although relative risk reduction decreased with age. On the contrary, a recent meta-analysis (Boyle et al., 2009) including 12 randomizedcontrolled trials (781 participants) conclude that probiotics are not a clinically effective treatment for eczema, although when given during early infancy for the prevention rather than the treatment of eczema the results for some probiotic strains are promising.

20 The effects that probiotics have on intestinal permeability, intestinal inflammation, microbiota composition and host immune responses may be insufficient to lead to the resolution of established eczema, but sufficient when used in early life for the prevention of such disease. One potential criticism of this meta-analysis is the low number of studies and the inclusion of heterogeneous studies using different probiotic strains. 
A recent study (Vliagoftis et al., 2008) evaluated the clinical evidence for the use of probiotics as a therapeutic modality for allergic rhinitis and asthma. Probiotics may have a beneficial effect in allergic rhinitis by reducing symptom severity and medication use, although, further good-quality studies are needed (Hatakka et al., 2000). Indeed,

5 Lactobacillus paracasei might improve the quality of life of adolescents with perennial allergic rhinitis (Peng et al 2005; Wang et al., 2004). To examine whether long-term consumption of fermented milk containing a specific Lactobacillus casei may improve the health status of children suffering from allergic asthma and/or rhinitis a randomized, prospective, double blind, controlled trial was conducted (Giovanini et al, 2007). In

10 children with rhinitis, the annual number of rhinitis episodes was lower in the intervention group, and also the mean duration of an episode of diarrhea was lower in the intervention group. While long-term consumption of fermented milk containing Lactobacillus casei may improve the health status of children with allergic rhinitis no effect was found in asthmatic children (Giovanini et al, 2007).

\section{Oral cavity}

The microflora of the oral cavity is comprised of more than 500 bacterial species (Dewhirst et al. 2009). The most prominent cariogenic bacterium is Streptococcus mutans, but many lactobacilli have cariogenic properties too. The composition of the

20 oral microflora is influenced by bacterial transfer at birth, the gastrointestinal flora, the composition of the saliva and by other factors. The long-term composition is remarkably stable and does not vary with individual meals. The main focus in the studies of potential oral probiotics has been on caries prevention, especially on the possibility of reducing the number of mutans streptococci when products containing certain probiotic strains are used (Haukioja et al., 2008). The caries bacteria colonize 
acidic environments, such as fissures and interdental spaces, and produce acids from fermentable sugars. On the contrary there was a suggestion that temporary colonization of a nonlactose and slow sugar fermenting bacteria, which are able to inhibit the caries pathogens, may prevent caries in children (Meurman et al., 1994, Nasse et al., 2001).

5 Other possible applications, like the reduction of the number of oral Candida or of microflora associated with oral malodour, have also been investigated.

The probiotic strains most studied in relation to the colonization of mutans streptococci are L. rhamnosus GG and L. reuteri ATCC 55730 (Ahola et al., 2002; Caglar et al., 2005, 2006 ; Meurman et al., 1995; Näse et al., 2001; Nikawa et al., 2001). In some

10 studies the use of products containing these bacteria has been found to reduce oral mutans streptococci (Näse et al., 2001; Nikawa et al., 2001; Caglar et al., 2006). L. rhamnosus GG can be found in the oral cavities of volunteers who are using products containing this bacterium (Yli-Knuuttila et al., 2006). Colonization by L. reuteri SD2112 has not been studied, but the products containing it do not increase the

15 lactobacillus counts in saliva (Canglar et al., 2006). Other probiotic strains have also been studied but with contradictory results: for example, a product containing Bifidobacterium DN-173010 seems to decrease the number of mutans streptococci in saliva (Canglar et al., 2005), but Montalto et al. (2004) did not find any effect of probiotic treatment on salivary counts.

20 Although several studies have shown that some probiotic bacteria may affect oral ecology, the mechanisms are largely unknown. A recent study (Haukioja et al., 2008) showed that probiotic bacteria that bound to saliva-coated hydroxyapatite reduced the adhesion of $S$. mutans but the inhibitory effect on the adherence of $S$. gordonii was lower. Salivary pellicle protein composition was modified by all the strains tested.

25 Further studies are needed on this filed to clarify the potential role of probiotics. 


\section{Skin diseases}

The normal human skin microflora is variable according to location and composed of a limited number of microbial types (Grice et al. 2009). The resident microbiota fills a

5 niche that could otherwise be colonized by pathogenic microorganisms that are aggressive and cause infection, either at the skin site or by transfer to other sites. Also, if the skin could be colonized by pathogens adopted for other sites (e.g. mucous membranes), and this could facilitate the spread of pathogens by offering them more dispersed routes.

10 As stated above, a significant improvement on the course of atopic dermatitis (Isolauri et al., 2000) and also, probiotics may be able to reduce the prevalence of atopic eczema in children at high risk for atopic diseases (Kalliomaki et al., 2001) have been described. The suggested mechanistic base of skin effects induced by probiotic strains is represented by changes in systemic immune responses. In particular, modulation of

15 specific T-cell subsets such as stimulation of TH1 cells in the gut mucosa which may subsequently influence immune responses in other tissues may play a role (Pohjavuori et al., 2004; Lammers et al., 2003, Prescott et al., 2005). In mice, oral administration of Lactobacillus casei reduced contact hypersensitivity to a hapten only in the presence of CD4+ T-cells, which control the size of the CD8+ effector pool (Chapat et al., 2004).

20 Nutritional supplementation of mice with Lactobacillus johnsonii provided protection of the skin immune system against ultraviolet B radiation-induced immunosuppressive effects (Gueniche et al., 2006). Similar effects have recently been described in a human in vivo study and it has been proposed that oral consumption of probiotic bacteria may represent a novel approach to protect the skin immune system against ultraviolet radiation (Bouilly et al.,2008). Another target for probiotics may be skin barrier 
function. A recent double-blind, randomized clinical study has shown that a 24-week skin nutrition intervention with a fermented dairy product in female volunteers having dry and sensitive, but otherwise healthy skin significantly reduced transepidermal water loss and thus improved stratum corneum barrier function compared to a placebo product

5 (Punch et al., 2008). It should be noted, however, that in addition to the probiotic strains (L. casei, Lactobacillus bulgaris and Streptococcus thermophilus) this dairy product contained a mixture of boretsch oil, green tea polyphenols and vitamin E and that skin barrier improvement may be at least in part due to these ingredients. In general, nonintestinal applications of probiotics are few and have thus far mainly been used for

10 the urogenital tract. There are currently only a very few studies pursuing a probiotic approach for the skin bacterial composition. Ouwehand et al. (2003) point out the difficulty to identify useful bacteria in view of the environmental conditions that may prevent colonization of skin with a probiotic strain.

\section{Obesity and Insulin Control}

The prevalence of obesity is rapidly increasing in Western society, constituting an important health issue. Obesity is the result of a positive imbalance between energy intake and energy expenditure over a long period of time and its related to the development of other diseases including diabetes and cardiovascular diseases among

20 others. Several genetic and environmental factors influence obesity (Hill et al., 1998; Jouret et al., 2007) which include diet, socioeconomic status, maternal obesity, rapid infancy weight gain, and decreased physical activity. In addition to all of these risks, some reports have suggested that the gut microbiota is an important factor affecting energy disposal and storage in adipocytes (Ley et al., 2005, Turnbaugh et al., 2006). 
Experimental research suggests that the nature and composition of the intestinal microbiota are altered in obesity (Ley et al., 2005; Turnbaugh et al., 2006). Lean individuals have more bacteroides type bacteria, while obese individuals have more Firmicutes such as clostridia in their intestinal microbiota (Ley et al., 2005; Turnbaugh

5 et al., 2006). It has been proposed that such bacterial composition lead to improved extraction of energy from the diet and enhanced energy storage in the adipose tissue (Turnbaugh et al., 2006).

In addition, a recent report showed that infants with high numbers of the Bifidobacterium and low numbers of Staphylococcus may be protected from excess 10 weight gain during later life (Kalliomaki et al., 2008). Since Bifidobacterium genus and species are prevalent in breast-fed infants, it has been suggested the protective role attributed to breast-milk against developing obesity later in life (Wabitsh 2000). In addition, it has been reported how diets based on a high intake of protein and/or low intake of carbohydrate or low fat consumption may alter microbial composition and

15 activity in the large intestine and thus impact on gut health (Cani et al., 2008, Duncan et al., 2007). Obese humans under dietary intervention, based on low carbohydrate intake and high protein intake, showed reductions in Bifidobacterium, and Roseburia spp. and clostridial cluster XIVa levels when carbohydrate intake was decreased, while no differences were detected in Bacteroides or other clostridial clusters (Duncan et al., 20 2007). Alterations in gut microbiota composition associated with obesity have also been shown to be reversible by oral transfer of the gut microbiota from lean mice to a germfree recipient (Turnbaugh et al., 2006 and 2008) or by administration of prebiotic substrates to animal models at least over short-term periods (Cani et al., 2007). Therefore, it seems likely that a combination of environmental (e.g. diet) and genetic 
factors contributes to defining unique combinations of bacteria within an individual, which could favor either an obese or lean phenotype.

Gut microbiota modulation via dietary strategies such as probiotics may offer new directions for preventive and therapeutic applications in reducing the risk of overweight

5 and obesity and also, metabolic-associated disorders. Lee et al (2006) investigated the anti-obesity effect of a $L$. rhamnosus strain that produces conjugated linoleic acid, in diet-induced obese mice. After 8 weeks of oral feeding, mice lost weight without reducing energy intake. In another study, Martin et al (2008) administered probiotic beverages to germ-free mice that had been conventionalized with human infant

10 microbiota showed that probiotic exposure resulted in distinct changes in the microbiome with associated metabolic alterations affecting energy, lipid, and aminoacid metabolism. The importance of these findings to energy homeostasis and overall health in humans remains to be determined; however, they suggest that probiotics can alter the dynamics of the entire gut microbiota and show that these molecular approaches can be

15 used to study the metabolic effects of probiotics on the host and the host's microbiome.

\section{Cognitive Function and Mental Health}

As indicated above the use of probiotics has been tested for the prevention and treatment of different intestinal and extra-intestinal diseases. However the application of

20 this approach to other health-related situations has not been assessed to date.

The potential role of probiotics on the gut-brain axis provides another interesting area for future research. Consumption of certain probiotic strains may affect cytokine patters and it has been shown that these molecules may affect cognitive functions (Wilson et al. 2002) and gut hormones and neuropeptides may affect the learning process (Diano et al.

25 2006). Mental problems, including schizophrenia, anxiety, stress or depression, are 
frequently associated with other disorders in which microbiota disturbances may play a role, such as ulcerative colitis, celiac disease, metabolic syndrome or IBS (Peet 2006). Also immune parameters have been found to be affected in these patients (Atanackovic et al. 2004, Barak 2006). It has been known for a long time that stress (Holdeman et al.

5 1976), can affect microbiota composition. Interestingly, a role of the microbiota in the development of the physiological system of stress response has been suggested (Sudo et al. 2004). In a recent animal study a Bifidobacterium infantis strain was found to inhibit pro-inflammatory responses, increase plasma levels of tryptophan, which is an important serotonergic precursor, and to reduce levels of some monoamine metabolites

10 in the brain, suggesting a potential antidepressant effect of the strain (Desbonet et al. 2008). Interestingly, in a trial with a fermented milk containing L. casei Shirota Benton and coworkers (2007) observed a potential improvement of mood in healthy subjects.

These facts open the possibility for the participation of gut microbiota in many apparently unrelated physiological processes and diseases. If this is the case

15 intervention with probiotics may have an impact on these patients.

\section{Inflammation of the joins}

Common genetic and immunologic mechanisms underlay the coincidence of inflammation in the bowel and the joints. A recent study reports the association between

20 bacterial gastroenteritis and the risk of developing arthritic symptoms (Garg et al. 2008) and aberrancies on the intestinal microbiota composition have been identified in arthritis (Benno et al. 1994, Malin et al. 1996) and spondylitis (Stebbings et al. 2002). Rheumatoid arthritis patients harbour bacteria capable of triggering arthritis (Toivainen 2003) and in fact, bacterial DNA has been found in synovial fluids of these patients 
(Moen et al. 2005). Thus, gut microbiota together with appropriate immune modulation constitute clear targets for probiotics in patients suffering arthropathies.

\section{Conclusion and future perspectives}

5 Recent advances have been made in the understanding of when to use probiotics and how they impact specific pathological states. Several evidences support the concept that probiotic may have benefits in a multitude of disorders and they exert health promoting effects. Clinical efficacy of specific probiotic strains has been demonstrated in some diseases as rotavirus's diarrhea, antibiotic associated diarrhea, irritable bowel syndrome

10 and food allergies. In addition, new evidences support the use of probiotics in the prevention and treatment of a number of diseases including atopic diseases, immune disorders, obesity, and diabetes. Further, recent clinical and nutritional studies have uncovered the function of specific strains in energy metabolism and thereby have opened up new angles on their use and exploitation. However, as these processes are

15 highly specific, it is important to characterize the properties the probiotic strains an in order to select the best strains or strain combinations for the target in question. Advances have prompted increase the interest of researchers and industry and new applications and targets are being discovered.

The rapid development of massive sequencing techniques is allowing the flourisment of 20 metagenomic studies. The increased knowledge on genomics, microbiota, and interactions nutrients-genes (nutrigenomics) promises to expand our understanding on the interactions between food and health allowing the development of probiotic dietary intervention strategies tailored for specific individuals or functions. This is increasing enormously our knowledge on the complex gut microbiota composition and activities and allowing to identify disturbances on microbiota related to human disease. In general 
any disorder in which an aberrant microbiota or an un-appropriate immune response may play a role are potential targets for probiotic intervention, even though they may not take place at gut level. Disturbances on the microbiota have been identified in other intestinal disorders, including diverticulitis (Colecchia et al. 2003), and extraintestinal

5 conditions, such as elderly people suffering severe frailty (van Tongeren et al. 2005). Patients with severe systemic inflammatory response syndrome showed lower levels of bifidobacteria and lactobacilli and higher levels of pathogenic microorganisms than healthy subjects (Shimizu et al. 2006). Reduced levels of bifidobacteria have also been shown in multiple sclerosis patients (Wagenfeld 1991). Although, so far probiotics have 10 not been tested in these settings, these studies indicate potential targets for the future development of probiotic products.

Some intestinal bacteria have been suggested for very specific targets. For instance Oxalobacter formigenes may have a potential role to play in the risk reduction for kidney stone formation. (Stewart et al. 2004). A Bacteroides strain, able to reduce 15 cholesterol, isolated from the gut microbiota of a subject with a high ability to reduce cholesterol to coprostanol has also been reported (Gerard et al. 2007). Such microorganisms may constitute the basis for future targeted probiotic developments. To this regard, other areas of the body different to the gastrointestinal and urogenital tracts, such as nasopharynx or skin may also provide areas for novel probiotic applications.

20 In any case the high strain-specificity of probiotics must always be taken into consideration. In fact, the strain-specific characteristics can be exploited to select more efficacious strains or for selecting probiotics for specific targets or populations. 


\section{ACKNOWLEDGEMENTS}

All authors participated in preparation of the manuscript. None of the authors have conflict of interests. This work was supported Consolider Fun-C-Food CSD2007-00063 from the Spanish Ministry of Science and Innovation. M.C.C. is the recipient of a

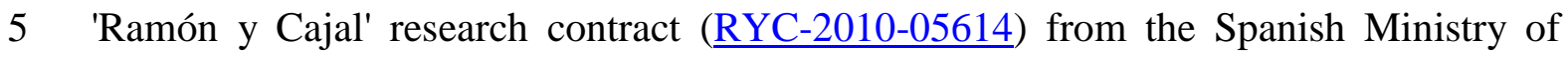
Science and Innovation, Spanish Government. 